\title{
Quasi-hydrostatic intracluster gas under radiative cooling
}

\author{
K. Masai ${ }^{1}$ and T. Kitayama ${ }^{2}$ \\ 1 Department of Physics, Tokyo Metropolitan University, Hachioji, Tokyo 192-0397, Japan \\ 2 Department of Physics, Toho University, Miyama, Funabashi, Chiba 274-8510, Japan \\ e-mail: kitayama@ph.sci.toho-u.ac.jp
}

Received 19 February 2004 / Accepted 23 March 2004

\begin{abstract}
Quasi-hydrostatic cooling of the intracluster gas is studied. In the quasi-hydrostatic model, work done by gravity on the inflow gas with $\mathrm{d} P \neq 0$, where $P$ is the gas pressure, is taken into account in the thermal balance. The gas flows in from the outer part so as to compensate the pressure loss of the gas undergoing radiative cooling, but the mass flow is so moderate and smooth that the gas is considered to be quasi-hydrostatic. The temperature of the cooling gas decreases toward the cluster center, but, unlike cooling flows with $\mathrm{d} P=0$, approaches a constant temperature of $\sim 1 / 3$ the temperature of the non-cooling ambient gas. This does not mean that gravitational work cancels out radiative cooling, but means that the temperature of the cooling gas appears to approach a constant value toward the cluster center if the gas maintains the quasi-hydrostatic balance. We discuss the mass flow in quasi-hydrostatic cooling, and compare it with the standard isobaric cooling flow model. We also discuss the implication of $\dot{M}$ for the standard cooling flow model.
\end{abstract}

Key words. galaxies: clusters: general - cooling flows

\section{Introduction}

The intracluster gas, i.e. hot gas in clusters of galaxies, which undergoes radiative cooling is thought to flow in toward the cluster center to maintain pressure equilibrium (see Fabian 1994 for a review and references therein). According to the standard cooling flow picture, the mass deposition rate $\dot{M}$ can be estimated from the differential luminosity. X-ray observations showed that $\dot{M}$ inferred from the surface brightness profile was over $100 M_{\odot} \mathrm{yr}^{-1}$ for so called cooling flow clusters. However, firm evidence has not been found for such a large amount of cooled gas that should be detected at longer wavelengths if it existed in the cluster core (e.g., Edge 2001).

ASCA and recent Chandra and XMM-Newton observations (e.g., David et al. 2001; Kaastra et al. 2001; Allen et al. 2001; Peterson et al. 2003) with better spatial and spectral resolution reveal, however, that $\dot{M}$ is smaller by an order of magnitude than what was estimated before from the surface brightness. Moreover, spectroscopic analysis shows that the gas temperature decreases toward the cluster center, as expected for cooling flows, but down to only half or one-third the temperature of the non-cooling ambient gas. This implies the lack of soft X-ray emission, or no appreciable contribution of lower temperature gas.

To explain the observations, various theoretical models have been proposed (see e.g., Böhringer et al. 2002; Peterson et al. 2003). Many of those consider the central active galaxy (with an active galactic nucleus (AGN)) as a heating source

Send offprint requests to: K. Masai,

e-mail: masai@phys.metro-u.ac.jp to compensate radiation loss (e.g., Churazov et al. 2001; Ruszkowski \& Begelman 2002; Kaiser \& Binney 2003). This idea is interesting from the aspect of history of activity (Reynolds et al. 2002), since many cooling flow clusters possess $\mathrm{cD}$ galaxies in their central regions. However, some fine tuning is required. While the radiative cooling rate is determined by the local values of temperature and density, the conductive or convective heating rate including hot bubbles from AGN depends on the scale length of the temperature or pressure as well. This could make the processes complicated, and the parameters such as diffusion coefficient or mixing length remain open (Narayan \& Medvedev 2001; Voigt et al. 2002).

In a standard analysis of X-ray data, an isobaric cooling flow model is applied to estimate $\dot{M}$ (Fabian 1994). However, analysis based on surface brightness and analysis based on spectrum give inconsistent results, as mentioned above, in spite of both being based on the same physical concept. This may suggest some problem underlying the standard cooling flow model. In the standard isobaric model, work done by gravity on the inflow gas is not properly taken into account. This is not an additional heating source but is naturally expected for the gas under gravity. If the inflow is induced and regulated smoothly by local radiative cooling under gravity, quasihydrostatic structure may be attained. Such a simple picture is thought to be a starting point for considering an alternative heat source.

In the present paper we examine quasi-hydrostatic intracluster gas undergoing radiative cooling, taking proper account of the work done by gravity. In Sect. 2 we present our 
quasi-hydrostatic cooling model. We compare our model with the standard isobaric cooling flow model in Sect. 3, and give concluding remarks in Sect. 4.

\section{Quasi-hydrostatic cooling model}

The internal energy $U$ of the gas changes as

$\mathrm{d} U=C_{V} \mathrm{~d} T=\mathrm{d}^{\prime} Q-P \mathrm{~d} \frac{1}{\rho}$

where $C_{V}$ is the specific heat at constant volume, and $T, \rho$ and $P$ are the gas temperature, density and pressure, respectively. The first and second terms on the right-hand side represent heat flowing into and work done on the gas, respectively. If the gas is flowing toward the cluster center, work by gravitational force would be done on the gas.

We consider a spherically symmetric structure. By using the EOS (equation of state) of an ideal gas and the hydrostatic balance equation

$\frac{1}{\rho} \mathrm{d} P=-\frac{G M_{r}^{*}}{r^{2}} \mathrm{~d} r$

Eq. (1) is rewritten for the quasi-hydrostatic intracluster gas, as

$\frac{5}{2} k \frac{M}{\mu \mathrm{m}} \mathrm{d} T=-L \mathrm{~d} t-\frac{G M_{r}^{*}}{r^{2}} M \mathrm{~d} r$.

Here $M(r)$ is a gas mass undergoing radiation loss at a rate $L(r)$; one can consider, for instance, a shell as $M(r)=4 \pi r^{2} \rho \Delta r . M_{r}^{*}$ is the gravitational mass contained in the radius concerned, $\mu$ the mean molecular weight, $m$ the proton mass, and other quantities have their usual meanings. The term "quasi-hydrostatic" does not mean static but is used for the steady state under gravity.

From Eq. (3) and the continuity equation,

$\dot{M}_{r}=-4 \pi r^{2} \rho \frac{\mathrm{d} r}{\mathrm{~d} t}$,

we obtain

$\dot{M}_{r}\left(\frac{5}{2} \frac{k}{\mu \mathrm{m}} \frac{\mathrm{d} T}{\mathrm{~d} r}+\frac{G M_{r}^{*}}{r^{2}}\right)=4 \pi r^{2} \rho \frac{L}{M}=4 \pi r^{2} n^{2} \Lambda$,

where $n=\rho / \mu \mathrm{m}$ is the number density and $n^{2} \Lambda$ the cooling rate per unit volume. $\dot{M}_{r}$ represents the gas mass flowing from the outer part into $r$ per unit time.

We assume that the intracluster gas is isothermal before cooling when virialized, i.e., $(3 / 2)(k T / \mu \mathrm{m}) \sim-\phi$ for the gravitational potential $\phi$ in the cluster (see e.g., Sarazin 1986). In such a case, the gravitational mass $M_{r}^{*}$, which includes the galaxies and the dark matter as well as the gas, can be replaced with the equilibrium (virial) temperature $T_{0}$, as

$\frac{G M_{r}^{*}}{r} \simeq \frac{3}{2} \sigma_{r}^{2}=\frac{3}{2} \beta \frac{k T_{0}}{\mu \mathrm{m}}$,

where $\sigma_{r}$ is the line-of-sight velocity dispersion of the galaxies in the cluster; $\beta$ is a parameter in the so-called $\beta$-model of intracluster gas. We consider that $M_{r}^{*}$ is large enough compared to the gas mass $M_{r}$ within $r$ and that the gas inflow hardly alters the gravitational potential.
Equation (5) with $T_{0}$ is expressed in the form

$$
\begin{aligned}
\frac{\mathrm{d} \ln T}{\mathrm{~d} \ln r} & =\frac{9}{5}\left[\frac{4 \pi}{3} r^{3} \frac{n^{2} \Lambda}{(3 / 2) k T} \frac{\mu \mathrm{m}}{\dot{M}_{r}}-\frac{1}{3} \beta \frac{T_{0}}{T}\right] \\
& =\frac{9}{5}\left[\frac{\tilde{M}_{r} / \tilde{t}_{\text {cool }}}{\dot{M}_{r}}-\frac{1}{3} \beta \frac{T_{0}}{T}\right],
\end{aligned}
$$

where, in the last expression,

$\tilde{M}_{r} \equiv \frac{4 \pi}{3} r^{3} \rho \quad$ and $\quad \tilde{t}_{\text {cool }} \equiv \frac{(3 / 2) n k T}{n^{2} \Lambda}$.

Hereafter the quantities with tildes represent those evaluated with the local values of density and temperature; $\tilde{M}_{r}$ and $\tilde{t}_{\text {cool }}$ are the mass of a uniform gas sphere and the cooling time, which are given by $\rho(r)$ and $T(r)$. Accordingly, $\tilde{M}_{r}$ represents the gas mass within $r$ that cools radiatively in the cooling time $\tilde{t}_{\text {cool }}$ given at $r$. Equations (5) or (7) shows the relation between variations $\mathrm{d} T$ and $\mathrm{d} r$ to satisfy Eq. (1) for the relation between $\dot{M}_{r}$ and $L$ under quasi-hydrostatic balance, i.e., hydrostatic balance (Eq. (2)) with a mass flow (Eq. (4)).

We consider that the gas inflow across $r$ is caused by radiative cooling within $r$ under quasi-hydrostatic balance at every $r$. While the gas of mass $\tilde{M}_{r}$ inside loses its pressure on a time scale $(\partial \ln P / \partial t)^{-1} \sim(\partial \ln T / \partial t)^{-1}=\tilde{t}_{\text {cool }}$, gas of temperature $T$ comes into the region at rate $\dot{M}_{r}$ to compensate the loss with the time scale $\tilde{t}_{\text {cool }}$. The inflow mass at $r$ during $\tilde{t}_{\text {cool }}$ is expressed approximately as

$\dot{M}_{r} \tilde{t}_{\text {cool }} \sim \frac{\mathrm{d} \tilde{M}_{r}}{\mathrm{~d} t} \tilde{t}_{\text {cool }} \sim \tilde{M}_{r}$

Therefore, we assume $\dot{M}_{r} \sim \tilde{M}_{r} / \tilde{t}_{\text {cool }}$ for a local quasi-static change at a given radius. This condition for observable quantities and its validity are discussed in detail later. It should be noted that $\tilde{M}_{r}$ differs from the gas mass $M_{r}$ contained within $r$, as it depends on the density profile $\rho(r)$. The cooling time of the gas $M_{r}$ is also different from $\tilde{t}_{\text {cool }}$. We mention later such global quantities in the discussion of the above condition.

The gas that undergoes radiative cooling has a temperature profile given by Eq. (7), while the ambient gas that is free from radiative cooling remains isothermal. We define a radius $r_{\mathrm{cool}}$ at which $\tilde{t}_{\text {cool }} \sim t_{\mathrm{H}}=H_{0}^{-1}$, the Hubble time. $\tilde{t}_{\text {cool }}<t_{\mathrm{H}}$ at $r<r_{\text {cool }}$, and $\tilde{t}_{\text {cool }}>t_{\mathrm{H}}$ at $r>r_{\text {cool }}$, because $\tilde{t}_{\text {cool }}$ becomes monotonically shorter toward the inner region. At $r>r_{\text {cool }} \sim 100 \mathrm{kpc}$, radiative cooling has little effect on the hydrostatic structure, and the gas temperature is nearly equal to the gravitational temperature. On the other hand, at $r<r_{\text {cool }}$ the effect of cooling becomes important. The temperature decreases and the gas is flowing inward, yet the gas is likely quasi-hydrostatic if the inflow is smooth and its speed is much slower than the local sound speed $\propto \sqrt{T}$, or more correctly, the ram pressure is small compared to the thermal pressure.

Thus, the temperature of the quasi-hydrostatic cooling gas begins to decrease from $T_{0}$ at $r_{\text {cool }}$, and approaches an asymptotic value given by

$T \sim \frac{1}{3} \beta T_{0} \frac{\dot{M}_{r}}{\tilde{M}_{r} / \tilde{t}_{\text {cool }}}$

toward the cluster center. In Fig. 1 we show a temperature profile given by Eq. (7) with $\dot{M}_{r} \sim \tilde{M}_{r} / \tilde{t}_{\text {cool }}$ at every $r$. For 


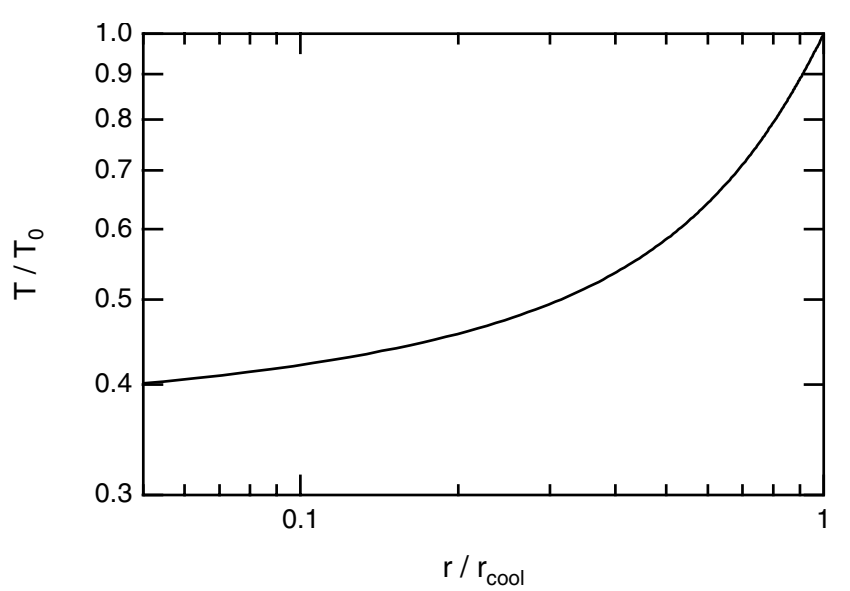

Fig. 1. The temperature of cooling quasi-hydrostatic gas, as a function of $r / r_{\text {cool }}$ for the case $\dot{M}_{r}=\beta^{-1} \tilde{M}_{r} / \tilde{t}_{\text {cool }}$. This corresponds, for instance, to $\dot{M}_{r}=\tilde{M}_{r} / \tilde{t}_{\text {cool }}$ for $\beta=1$ and to $\dot{M}_{r}=1.5 \tilde{M}_{r} / \tilde{t}_{\text {cool }}$ for $\beta=2 / 3$.

$\dot{M}_{r}=\beta^{-1} \tilde{M}_{r} / \tilde{t}_{\text {cool }}$, the temperature decreases as $T \propto r^{6 / 5}$ at $T \sim T_{0}$, as $T \propto r^{3 / 5}$ at $T \sim(1 / 2) T_{0}$, and eventually becomes $\sim(1 / 3) T_{0}$. In practice, at the cluster center, the gas accumulates and cools further to a lower temperature. It is likely that at $T \lesssim T_{0}\left(r \lesssim r_{\text {cool }}\right)$ the temperature is somewhat less steep than $T \propto r^{6 / 5}$ and becomes flatter outward so that the temperature connects smoothly with $T \sim T_{0}$ at $r \sim r_{\text {cool }}$. If electron conduction works, the temperature would vary more gradually at $0.5 \lesssim r / r_{\text {cool }} \lesssim 1$.

We examine the density profile of the gas in quasihydrostatic cooling considered here. For simplicity, we express the density and temperature profiles as $\rho \propto r^{\alpha}$ and $T \propto r^{\eta}$, where $\eta$ is given by Eq. (7). Since

$\tilde{M}_{r} \propto r^{3+\alpha}$ and $\tilde{t}_{\text {cool }} \propto r^{-\alpha+\eta(1-\xi)}$

with $\Lambda \propto T^{\xi}$, we have

$\frac{\tilde{M}_{r} / \tilde{t}_{\mathrm{cool}}}{\dot{M}_{r}} \propto r^{1+\alpha-\eta(1-\xi)-\zeta}$

with the inflow velocity $\propto r^{\zeta}$. Here $1-\xi>0$ in the temperature range of interest and it is likely $\zeta<0$ for smooth inflow. For a quasi-static change at given $r$, that is, the condition $\dot{M}_{r} \sim$ $\tilde{M}_{r} / \tilde{t}_{\text {cool }}$ means

$1+\alpha-\eta(1-\xi)-\zeta \sim 0$

with a small value of $|\zeta|\left(=r / L_{v}\right)$ compared to unity, where $L_{v}$ is the scale length of the inflow velocity.

Thus, when the gas is cooling with quasi-hydrostatic balancing, the density varies as

$\rho \propto r^{-1+\eta(1-\xi)+\zeta}$,

roughly as $\rho \propto r^{-0.5}$ at $r \lessgtr r_{\text {cool }}$ and approaches $\propto r^{-1}$ for $\eta \rightarrow 0$ toward the cluster center. Note that the local cooling time is independent of the temperature/density profile,

$\tilde{t}_{\text {cool }} \propto r^{1-\zeta}$,

and varies roughly as $\tilde{t}_{\text {cool }} \propto r$. Inversely, if observations show $\tilde{t}_{\text {cool }} \propto r$, this implies $-\alpha+\eta(1-\xi) \sim 1$ and therefore the validity of the assumption $\dot{M} \sim \tilde{M}_{r} / \tilde{t}_{\text {cool }}$ at every $r$ or $\left(\tilde{M}_{r} / \tilde{t}_{\text {cool }}\right) / \dot{M} \sim$ constant. A recent report on 16 clusters observed by Chandra (Voigt \& Fabian 2004) seems to suggest $\tilde{t}_{\text {cool }} \propto r$. These properties as well as the temperature profile predicted by the quasihydrostatic cooling model with $\dot{M}_{r} \sim \tilde{M}_{r} / \tilde{t}_{\text {cool }}$ can be confirmed by observations. The gas mass $M_{r}$ contained within $r$ is larger than $\tilde{M}_{r}$ for the density profile with $\alpha<0$. With $t_{\text {cool }}$ being the global cooling time of the gas $M_{r}$, as

$M_{r}=\int 4 \pi r^{2} \rho \mathrm{d} r \sim \frac{3}{3+\alpha} \tilde{M}_{r}$

and

$t_{\mathrm{cool}}=\frac{\int 4 \pi r^{2}(3 / 2) n k T \mathrm{~d} r}{\int 4 \pi r^{2} n^{2} \Lambda \mathrm{d} r} \sim \frac{3+2 \alpha+\eta \xi}{3+\alpha+\eta} \tilde{t}_{\mathrm{cool}}$,

the difference between $M_{r} / t_{\text {cool }}$ and $\tilde{M}_{r} / \tilde{t}_{\text {cool }}$ is estimated to be within a factor of $\sim 2$.

While the gas is cooling, $\dot{M}_{r}$ is coming in from the outer part so as to maintain the quasi-hydrostatic balance. Such a flow is not very drastic, since the gas is cooling monotonically and thereby the inflow is smooth. Thus, the mass flow rate follows

$\dot{M}_{r}=\frac{6}{5} \tilde{L}_{r} \frac{\mu \mathrm{m}}{k T}\left(\frac{\mathrm{d} \ln T}{\mathrm{~d} \ln r}+\frac{3}{5} \beta \frac{T_{0}}{T}\right)^{-1}$,

where $\tilde{L}_{r} \equiv(4 \pi / 3) r^{3} n^{2} \Lambda$ is given by the density and temperature at $r . \dot{M}_{r}$ is in the range $(2 / 3-2) \cdot \tilde{L}_{r}\left(\mu \mathrm{m} / k T_{0}\right)$ for $T_{0}$ and does not vary very much through the flow (see Sect. 4). For $\dot{M}_{r} \sim \tilde{M}_{r} / \tilde{t}_{\text {cool }}$, Eq. (16) reduces to

$$
\begin{aligned}
\dot{M}_{r} \sim & \frac{2}{3} \tilde{L}_{r} \frac{\mu \mathrm{m}}{k T} \\
\sim & 8.4\left(\frac{r_{\mathrm{cool}}}{100 \mathrm{kpc}}\right)^{3}\left(\frac{n}{10^{-3} \mathrm{~cm}^{-3}}\right)^{2}\left(\frac{k T_{0}}{\mathrm{keV}}\right)^{-1} \\
& \times\left(\frac{\Lambda}{10^{-23} \mathrm{erg} \mathrm{s}^{-1} \mathrm{~cm}^{3}}\right) M_{\odot} \mathrm{yr}^{-1}
\end{aligned}
$$

where $\mu=0.61$ is assumed.

For the values of typical cooling flow clusters, the mass flow rate may be $\sim 100 M_{\odot} \mathrm{yr}^{-1}$. The local inflow rate $\dot{M}_{r}$ does not mean the mass deposition rate (see Sect. 3) but merely gives an upper limit on the cooled mass in the cluster. Equations (16) or (17) means that following the change in $T$ (Eqs. (3) and (5)) $\dot{M}_{r}$ increases with increasing $\tilde{L}_{r}$. This is reasonable if the mass inflow is induced by radiation loss; $\dot{M}_{r}$ is not simply proportional to $\tilde{L}_{r}$ but depends also on $T$. The dependence in Eq. (17) should be compared with that in the standard isobaric cooling flow model, which is discussed in the following section.

\section{Comparison with isobaric cooling flow model}

In the quasi-hydrostatic model, work done by gravity on the intracluster gas is taken into account. The pressure is given by Eq. (2), and therefore $\mathrm{d} P \neq 0$ in this model. In comparison with quasi-hydrostatic cooling, we discuss isobaric cooling which is often applied to estimate the mass flow rate from X-ray observations. 
For given mass $M$, with $\mathrm{d} P=0$, Eq. (1) gives

$\frac{5}{2} k \frac{M}{\mu \mathrm{m}} \mathrm{d} T=\mathrm{d}^{\prime} Q=-L \mathrm{~d} t$.

This is nothing but the definition of the specific heat $C_{P}=$ $\left(\mathrm{d}^{\prime} Q / \mathrm{d} T\right)_{P}$ at constant pressure. Equation (19) simply expresses that for mass $M$ the temperature decreases with time due to radiation loss under $\mathrm{d} P=0$. The density must increase to maintain the pressure equilibrium, and the cooling time becomes shorter as $\propto T^{2} / \Lambda$. Thus, the gas cools and flows in increasingly toward the cluster center.

Equation (19) may be compared with the expression for the standard cooling flow model (Fabian 1994),

$\frac{5}{2} k \frac{\dot{M}_{\mathrm{CF}}}{\mu \mathrm{m}} \mathrm{d} T=\mathrm{d} L$,

where $\mathrm{d} T$ is defined for a change from $T$ to $T-\mathrm{d} T$ (Johnstone et al. 1992; Fabian 1994), i.e. d $T>0$ for cooling, and corresponds to the variation $-\mathrm{d} T$ in the present paper. So that, instead of the above equation, we consider

$\frac{5}{2} k \frac{\dot{M}_{\mathrm{CF}}}{\mu \mathrm{m}} \mathrm{d} T=-\mathrm{d} L$

as the standard cooling flow model. With increasing radiation loss $(\mathrm{d} L>0)$, the temperature decreases $(\mathrm{d} T<0)$ for given $\dot{M}_{\mathrm{CF}}(>0)$; otherwise, if $\dot{M}_{\mathrm{CF}}<0$ were the case, the temperature would increase for $\mathrm{d} L>0$.

According to the standard cooling flow model (Fabian 1994), $\mathrm{d} L$ in Eq. (20) is the differential luminosity defined as $\mathrm{d} L=(\mathrm{d} L / \mathrm{d} T) \mathrm{d} T$. Therefore, Eq. (20) is not a derivative of Eq. (19). This implies that Eq. (20) comes from some other concept than $\mathrm{d} P=0$ in Eq. (1). $\dot{M}_{\mathrm{CF}}$ may be interpreted as the gas mass that is cooling and dropping out of the flow per unit time (see Wise \& Sarazin 1993) or the mass deposition rate (Johnstone et al. 1992).

For the enthalpy of the cooling gas of given $T$, with $\mathrm{d} P=0$ we may have

$\frac{5}{2} k \frac{\dot{M}}{\mu \mathrm{m}} T \sim-L$,

consistently with Eq. (19) for given $M$. Equation (21) means $\dot{M}<0$ (cf. $\dot{M}_{\mathrm{CF}}>0$ ) or that the gas of temperature $T$ decreases at a rate $\propto L$. The lost gas must be so cool, much cooler than $T$, that it no longer contributes to $L$ at all.

Suppose that the intracluster gas has temperature $T$ to emit $L$. With $M_{\mathrm{d}}$ being the cooled mass, it increases at a rate roughly as $\dot{M}_{\mathrm{d}} \sim-\dot{M}>0$ in the cluster. So that, for the mass deposition rate $\dot{M}_{\mathrm{d}}$, we have a global relation

$\frac{5}{2} k \frac{\dot{M}_{\mathrm{d}}}{\mu \mathrm{m}} T \sim L$,

where the left-hand side does not mean the enthalpy of the cooling gas; remember that the gas of $M_{\mathrm{d}}$ should be too cool $(\ll T)$ to be responsible for $L$. Even if $\dot{M}_{\mathrm{CF}}$ is considered to be $\dot{M}_{\mathrm{d}}$, however, it is unlikely that Eq. (22) reduces to Eq. (20) for thermodynamical variation $\mathrm{d} T$. As readily seen, Eq. (22) gives $\dot{M}_{\mathrm{d}} \propto L / T$, while $\dot{M}_{\mathrm{CF}} \propto \mathrm{d} L / \mathrm{d} T$ in Eq. (20). Accordingly, the standard cooling flow model would yield an $\dot{M}_{\mathrm{CF}}$ that traces $\mathrm{d} \Lambda / \mathrm{d} T$, which is determined by atomic processes regardless of the thermal properties of the intracluster gas, as discussed below.

Equation (20) means that $\dot{M}_{\mathrm{CF}}$ is proportional directly to $-\mathrm{d} L / \mathrm{d} T$, because $\mathrm{d} L$ is the variation with $T$ in the standard cooling flow model. Considering $L \propto \rho^{2} \Lambda(M / \rho) \propto \rho \Lambda M$ with $P=$ const. $\equiv P_{0}$, we can express the luminosity in the form

$L=\frac{M}{\mu \mathrm{m}} \frac{\Lambda}{k T} P_{0}$.

Thus, Eq. (20) can be rewritten as

$$
\begin{aligned}
\frac{5}{2} k \frac{\dot{M}_{\mathrm{CF}}}{\mu \mathrm{m}} & =-\frac{\mathrm{d} L}{\mathrm{~d} T} \\
& =\frac{L}{T}\left(1-\frac{\mathrm{d} \ln \Lambda}{\mathrm{d} \ln T}\right) \\
& \sim \frac{5}{2} k \frac{\dot{M}_{\mathrm{d}}}{\mu \mathrm{m}}\left(1-\frac{\mathrm{d} \ln \Lambda}{\mathrm{d} \ln T}\right),
\end{aligned}
$$

where $1-\mathrm{d} \ln \Lambda / \mathrm{d} \ln T>0$ in the temperature range of interest. With decreasing $T$, the sign of $\mathrm{d} \ln \Lambda / \mathrm{d} \ln T$ changes to be negative at $k T \sim 2 \mathrm{keV}$, below which line cooling dominates over bremsstrahlung. While $\dot{M}_{\mathrm{CF}} \sim 0.5 \dot{M}_{\mathrm{d}}$ at $k T \gtrsim 2 \mathrm{keV}$, $\dot{M}_{\mathrm{CF}} \sim 1.5 \dot{M}_{\mathrm{d}}$ at $k T \lesssim 2 \mathrm{keV}$, on account of the factor $(1-\mathrm{d} \ln \Lambda / \mathrm{d} \ln T)$. Consequently, $\dot{M}_{\mathrm{CF}}$ would vary sensitively to local minima/maxima in $\Lambda(T)$.

Equation (23) has been applied to estimate the mass deposition rate from the soft X-ray luminosity (e.g. Peterson et al. 2003). In a limited photon-energy range, the $T$ dependence of $\Lambda$ becomes complicated because of the presence of lines. Therefore, observations with different energy bands could derive different values of $\dot{M}_{\mathrm{CF}}$, or spectral analyses could derive values different from each other. In any case, $\dot{M}_{\mathrm{CF}}$ of the standard cooling flow model seems not to give a reasonable representation of the mass deposition rate due to radiation loss $\mathrm{d}^{\prime} Q=-L \mathrm{~d} t$. If the luminosity $L$ of a cluster is well accounted for by emission of the intracluster gas of nearly isothermal temperature $T$, Eq. (22) would give roughly the isobaric deposition rate of the cooled-down mass in the cluster.

\section{Discussion and remarks}

In the quasi-hydrostatic model, we consider the ideal condition that a change is quasi-static. The pressure loss due to radiative cooling is immediately compensated by continuous, smooth, spherical mass inflow from the outer part. Neither turbulence nor overshooting occurs during the process. The inflow is so moderate as not to disturb the hydrostatic balance significantly. Thus, if the local inflow rate is perfectly controlled by the local cooling rate, the gas is quasi-hydrostatic and $\dot{M}_{r} \sim \tilde{M}_{r} / \tilde{t}_{\text {cool }}$ is attained; we will investigate this point further by means of hydrodynamical calculations elsewhere.

As mentioned in Sect. 2, "quasi-hydrostatic" means not static but steady-state. Accordingly, although the temperature of the cooling gas approaches a constant value toward the cluster center (Fig. 1), this does not mean that gravitational work can cancel out radiative cooling. This temperature profile is no 
more than a result of the steady inflow to maintain the hydrostatic balance. It should be noted that the gravitational potential or $M_{r}^{*}$ is assumed to be little affected by the gas inflow.

At the cluster center (or close to a cD galaxy) where the inflow mass is accumulating, the cooling time can be too short for the actual flow rate to follow the cooling rate of the gas inside, i.e. $\tilde{M}_{r} / \tilde{t}_{\text {cool }}>\dot{M}_{r}$. Then the quasi-hydrostatic balance would break, and the gas temperature inside decreases below $\sim(1 / 3) \beta T_{0}$, yet the temperature in the steady flow region will continue to follow Eq. (7).

In the opposite case, if the cooling rate at a given radius is smaller than the inflow rate as $\tilde{M}_{r} / \tilde{t}_{\text {cool }}<\dot{M}_{r}$, the flow rate $\dot{M}_{r}$ would decrease inward and the temperature approaches a constant value higher than $\sim(1 / 3) \beta T_{0}$ toward the cluster center. This may be the case when cooling in the core is not yet very significant or when some heating works against local radiative cooling so that $\tilde{t}_{\text {cool }}$ is effectively longer than given by Eq. (8).

The gas gains momentum during inflow. In the quasi-static model this is included into Eq. (2), which determines the hydrostatic structure in cooperation with EOS. This is valid as long as the momentum flux is small compared to the thermal pressure. In practice, however, particularly in the region where the temperature approaches a constant value (see Fig. 1), the inflow gas may partly overshoot because of a fluctuation about $\dot{M}_{r} \sim \tilde{M}_{r} / \tilde{t}_{\text {cool }}$. This may lead to instability and result in a break in the spherically symmetric structure. The resultant dense part cools more rapidly, but the gas remains quasihydrostatic on the whole if the momentum flux is still small on average.

Within the context of quasi-hydrostatic cooling, the gas inflow must be mild at every radius. However, if the flow evolved so that the momentum flux would become considerable, though still subsonic, the quasi-hydrostatic balance would fail unless some force works against the inertia. In such a case, for the gas to be quasi-hydrostatic, some momentum flux not in the form of the thermal pressure by heat may be needed against the inflow.

Observations by coming missions can examine the radial profiles predicted by the quasi-hydrostatic cooling model for several quantities, such as local cooling time $\tilde{t}_{\text {cool }}$, mass flow rate $\dot{M}_{r}$ or local luminosity $\tilde{L}_{r}$, and possibly inflow velocity as well as the density and temperature. The observed temperature profiles can be understood invoking quasi-hydrostatic cooling, though some momentum/heat source may be working additionally. The simple quasi-hydrostatic model is expected to give a basis for understanding the thermal properties of the intracluster gas under gravity and for advanced study using hydrodynamics codes.

Acknowledgements. The authors would like to thank Naomi Ota for discussion about the structure of intracluster gas and her helpful comments. Part of this work was supported by the Grant in Aid (14740133, 15037206) for Scientific Research of the Ministry of Education and Science in Japan.

\section{References}

Allen, S. W., Schmidt, R. W., \& Fabian, A. C. 2001, MNRAS, 328, L37

Böhringer, H., Matsushita, K., Churazov, E., Ikebe, Y., \& Chen, Y. 2002, A\&A, 382, 804

Churazov, E., Sunyaev, R., Forman, W., \& Böhringer, H. 2001, ApJ, 554,261

David, L. P., Nulsen, P. E. J., McNamara, B. R., et al. 2001, ApJ, 557, 546

Edge, A. C. 2001, MNRAS, 328, 762

Fabian, A. C. 1994, ARA\&A, 32, 227

Johnstone, R. M., Fabian, A. C., Edge, A. C., \& Thomas, P. A. 1992, MNRAS, 255, 431

Kaastra, J. S., Ferrigno, C., Tamura, T., et al. 2001, A\&A, 365, L99

Kaiser, C., \& Binney, J. 2003, MNRAS, 338, 837

Narayan, R., \& Medvedev, M. V. 2001, ApJ, 562, L129

Peterson, J. R., Kahn, S. M., Paerels, F. B., et al. 2003, ApJ, 590, 207

Reynolds, C. R., Heinz, S., \& Begelman, M. C. 2002, MNRAS, 332, 271

Ruszkowski, M., \& Begelman, M. C. 2002, ApJ, 581, 223

Sarazin, C. L. 1986, Rev. Mod. Phys., 58, 1

Voigt, L. M., Schmidt, R. W., Fabian, A. C., Allen, S. W., \& Johnstone, R. M. 2002, MNRAS, 335, L7

Voigt, L. M., \& Fabian, A. C. 2004, MNRAS, 347, 1130

Wise, M. W., \& Sarazin, C. L. 1993, ApJ, 415, 58 